JINR E2 - 95-203

ZU - TH- $4 / 95$

\title{
ON ADMISSIBLE GAUGES FOR CONSTRAINED SYSTEMS
}

\author{
S. A. Gogilidze, ${ }^{1}$ A. M. Khvedelidze, [l V. N. Pervushin \\ Joint Institute for Nuclear Research , 141980, Dubna, Russia
}

\begin{abstract}
The gauge - fixing and gaugeless methods for reducing the phase space in the generalized Hamiltonian dynamics are compared with the aim to define the class of admissible gauges .

In the gaugeless approach, the reduced phase space of a Hamiltonian system with the first class constraints is constructed locally, without any gauge fixing, using the following procedure: abelianization of constraints with the subsequent canonical transformation so that some of the new momenta are equal to the new abelian constraints. As a result the corresponding conjugate coordinates are ignorable ( nonphysical ) one while the remaining canonical pairs corresponds to the true dynamical variables. This representation for the phase space prompts us the definition of subclass of admissible gauges - canonical gauges as functions depending only on the ignorable coordinates. A practical method to recognize the canonical gauge is proposed .
\end{abstract}

${ }^{1}$ Permanent address: Tbilisi State University, 380086, Tbilisi, Georgia.

${ }^{2}$ Permanent address: Tbilisi Mathematical Institute, 380093, Tbilisi, Georgia. Electronic address: khved@theor.jinrc.dubna.su 


\title{
ON ADMISSIBLE GAUGES FOR CONSTRAINED SYSTEMS
}

\author{
S. A. Gogilidze, A. M. Khvedelidze, V. N. Pervushin
}

\section{Contents}

\begin{tabular}{|cc}
\hline Introduction & $\mathbf{1}$
\end{tabular}

II Phase space of the Hamiltonian system with constraints $\quad \mathbf{4}$

A Definition of the reduced phase space . . . . . . . . . . . . . . . . . . . . 4

B Reduced phase space in the gauge fixing method . . . . . . . . . . . . . . . . 4

B.1 Dirac's scheme without constraint resolution . . . . . . . . . . . . . . 4

B.2 Faddeev's scheme with constraint resolution . . . . . . . . . . . . . . 6

C Construction of the reduced phase space without gauge fixing via the "generalized canonical transformation" . . . . . . . . . . . . . . . . . . . . . 7

C.1 Abelian constraints . . . . . . . . . . . . . . . . . . . . . 7

C.2 Non-Abelian constraints . . . . . . . . . . . . . . . . . . . . . . 9 9

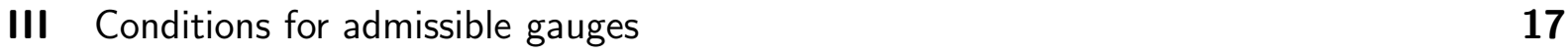

\begin{tabular}{|l|l}
\hline V Christ and Lee model & $\mathbf{1 9}$
\end{tabular}

A Abelian Christ \& Lee model . . . . . . . . . . . . . . . . . . . . . . . . . . . . . 19

B Non - Abelian Christ \& Lee model . . . . . . . . . . . . . . . . . . . . . . . 23

\begin{tabular}{lr}
\hline Concluding remarks & 24 \\
\hline
\end{tabular}

\begin{tabular}{lr}
\hline VI Acknowledgments & 24
\end{tabular} 


\section{Introduction}

It is the purpose of this note to discuss the problem of constructing of "true dynamical degrees of freedom" in the degenerate theories with first class constraints with the aim to obtain a constructive definition of admissible gauges.

A general method for describing degenerate theories starts by introducing the gauge fixing condition ( gauge fixation ) for elimination of nonphysical degrees of freedom [1] - [3]. Afterwards, there are two significantly different ways for reduction in number of degrees of freedom : explicit and implicit. The explicit method is straightforward - one must deal in an explicit way only with the physical variables while nonphysical ones are completely excluded from the consideration by gauge fixation. For the Abelian gauge theories this method works, one can always find the gauge invariant variables and identify them with physical ones while the rest variables can be dropped out via the gauge conditions. A problem arises, for example, in the Yang - Mills theory and gravity, where the true dynamical degrees of freedom are hidden due to the non - Abelian character of theories. The problems concerning the determination of the physical degrees of freedom in the Yang - Mills theory have been discussed by many authors (see e.g. [4] - [11]). A lot of attempts have been undertaken to realize the explicit separation of the nonphysical sector from the physical one. However, there still remain open questions and as a rule in the practical calculations we deal with the implicit form of gauge fixation. In this case, the general method [1], providing the restriction on the canonical variables due to constraints (including gauge fixing) consist in the determination of the modified symplectic structure of the phase space with the help of Dirac 's bracket [2] . In this method, one retains all dynamical variables and merely changes their Poisson brackets, which corresponds to the effective reduction in the number of degrees of freedom. As a result, one could not in general indicate the coordinates of the reduced system corresponding to the "true dynamical degrees of freedom ". However, to attain the correct results, one must be sure that the gauge fixing condition allows one to eliminate nonphysical degrees of freedom and fix the physical one in a unique way ( up to canonical transformation ) without any restriction on them. One can ask the question : are there some requirements to the gauge fixing conditions that quarantee such a correct description.

It is important to note that there are two sides of this question : global and local [3] . The well known manifestation of the problem of determining of globally admissible gauges is the so called Gribov ambiguity for the Yang - Mills theory. [12]. Singer's no - go theorem for

gauge fixing [13] rises questions about the generalization of the usual procedure of reducing 
degrees of freedom based on local manipulations. However, as we want to emphasize, at least for a local procedure it is necessary to clarify the reduction scheme. Just this is the goal of the present paper.

According to Dirac's prescription for generalized Hamiltonian systems, the reduction in the number of degrees of freedom consist in the elimination of first class constraints

$$
\varphi_{\alpha}(p, q)=0
$$

by introducing some new "gauge constraints" into the theory

$$
\chi_{\alpha}(p, q)=0
$$

and by replacing of the Poisson bracket by the Dirac one [1]]. The gauge functions $\chi$ are arbitrary functions of coordinates and momenta. There is only one requirement on the gauge fixing condition - nonvanishing of the Faddeev - Popov determinant on the constraint ( including gauge fixing ) shell

$$
\left.\operatorname{det}\left\|\left\{\chi_{\alpha}(p, q), \varphi_{\beta}(p, q)\right\}\right\|\right|_{\varphi=0, \chi=0} \neq 0
$$

However, it is known that (1.1]) is only a necessary condition for the gauge constraints [3]. There are examples of gauge constraints fulfilling (1.1), but as a result of reduction we get some restriction on physical sector - some type of overconstraining. For explanation, let us consider the simple case of QED with constraints

$$
\varphi_{1}=\pi_{0}, \quad \varphi_{2}=\partial_{i} \pi_{i}
$$

If one chooses the following gauge

$$
\chi_{1}=A_{0}=0, \quad \chi_{2}=A_{3}=0
$$

then the Faddeev - Popov matrix $\left\{\chi_{\alpha}, \varphi_{\beta}\right\}$ has a non - singular determinant on appropriate function space. But it is easy to state that this gauge leads to overconstraining of the system . Indeed, according to the essence of gauge fixing - to get rid of some degrees of freedom, the gauge - fixing condition allows one to determine in a unique manner the gauge transformation function from this gauge fixing conditions [14] :

$$
\begin{aligned}
& A_{0}^{\lambda} \equiv A_{0}+\partial_{0} \lambda=0 \\
& A_{3}^{\lambda} \equiv A_{3}+\partial_{3} \lambda=0 .
\end{aligned}
$$

It is obvious that there is a unique solution to these equations with respect to $\lambda$ if the integrability condition is satisfied

$$
\partial_{3} A_{0}-\partial_{0} A_{3} \equiv 0
$$


Thus, we obtain the restriction on the physical variable, the third component of the electric field

$$
\pi_{3}=0
$$

and get the overconstraining of QED. ( see e.g [15], [16] ).

So, to be sure that we are free from some incompatibility, it would be ideal if one could pick out directly the degrees of freedom (whitch are unconstrained) that have to be dropped out from a set of canonical pairs and then one would work in the reduced phase space. In other words, to get some information on restriction of gauge conditions, it is necessary to deal with some scheme that allows us to determine the reduced dynamics in a gaugeless manner and then to compare it with gauge fixing method. Fortunately, there is an elegant method of reducing the number of degrees of freedom known for systems of equations in involution [17], [18]. Levi- Civita has proposed the way of using the invariant relations (constraints in modern notations ) to reduce the order of the canonical system by passing to new canonical variables. As a result of the application of this scheme [19] - [21], the new canonical variables in the reduced system describe the allowed dynamics in terms of physical variables. It should be noted that for a direct application to the non - Abelian theory and gravity there is a serious obstacle. In this case, before carring out the canonical transformation to new variables, the constraints must be replaced by the equivalent set of constraints that form a canonical functional group. There is a general proof of a possibility of such a replacement [22] - [25], but the problem is to determine this new set in a constructive fashion. Nevertheless, this gaugeless scheme allows one to obtain some restriction on gauge fixing conditions .

In the present paper, based on the gaugeless scheme of reduction of the phase space we suggest in a constructive manner a certain subclass of admissible gauges (canonical gauges) for gauge theories with a first class constraints which can be exploited in the gauge fixing method. One can note a simple condition for gauge fixing functions which can serves a criterion for belonging to the class of canonical gauges - the requirement of vanishing the Dirac bracket of matrix $\Delta_{\alpha \beta}=\left\{\chi_{\alpha}, \varphi_{\beta}\right\}$ with the canonical Hamiltonian on the constraint (including gauge fixing) shell

$$
\left.\left\{\Delta_{\alpha \beta}(p, q), H_{C}(p, q)\right\}_{D}\right|_{\varphi=0, \chi=0}=0
$$

This article is organized as follows. In the first part of this paper we shall briefly describe Dirac's gauge fixing method and the gaugeless one. Section II is devoted to the definition of admissible gauges based on the canonical equivalence between two methods. In the last section, the general consideration of the admissible gauges is exemplified by Christ and Lee model [26. 


\section{Phase space of the Hamiltonian system with constraints}

For the sake of simplicity, as usual we will discuss the main ideas using mechanical system, i.e. system with a finite number of degrees of freedom, with having in mind that the transition to a field theory involves additional features connected with boundary effects.

\section{A Definition of the reduced phase space}

Suppose that in the system with finite number of degrees of freedom we have the following first class constraints

$$
\begin{aligned}
\varphi_{\alpha}(p, q) & =0 \\
\left\{\varphi_{\alpha}(p, q), \varphi_{\beta}(p, q)\right\} & =f_{\alpha \beta \gamma}(p, q) \varphi_{\gamma}(p, q) .
\end{aligned}
$$

This means that the dynamics of our system is constrained on a certain submanifold of the total phase space which is defined by the constraints $\varphi_{\alpha}(p, q)$ in (2.3). Further, we will symbolize by notation $\Gamma_{c}$ this $2 n-m$ - dimensional submanifold of the total phase space $\Gamma(\operatorname{dim}\|\Gamma\|=2 n) \Gamma_{c} \subset \Gamma$. For definition of the reduced or physical phase space we need the notion of physical variable. According to Dirac: A dynamical variable $F$ is of physical importance only if its Poisson bracket with any constraints gives another constraint [27]

$$
\left\{F(p, q), \varphi_{\alpha}(p, q),\right\}=d_{\alpha \gamma}(p, q) \varphi_{\gamma}(p, q)
$$

Such a dynamical variable is called a physical variable. According to this definition, in the process of evolution a physical variable does not abandon some subspace of $\Gamma_{c}$. Indeed [28], [29], if one consider, (2.4) as a set of $m$ first order linear differential equations for $F$, than due to the integrability condition (2.3) this function can be completely determined by its values in the $2(n-m)-m$ submanifold of its initial conditions. Thus, observables are functions on the socalled reduced phase submanifold $\Gamma^{*} \Gamma^{*} \subset \Gamma_{c} \subset \Gamma$ spanned by some physical coordinates $Q_{i}^{*}, P_{i}^{*}(i=1, \ldots, 2(n-m))$. Below we will discuss alternative schemes of construction of the reduced phase space: gauge - fixing and gaugeless methods.

\section{B Reduced phase space in the gauge fixing method}

\section{B.1 Dirac's scheme without constraint resolution}

Let us briefly describe the general principles of the introduction of gauge fixing constraints on canonical variables in a Hamiltonian theory. This general procedure to deal with physical variables was proposed by Dirac for the application to the Hamiltonian theory of gravitation [1]. 
The generalized Hamiltonian dynamics is described by the extended Hamiltonian

$$
H_{E}(p, q)=H_{C}(p, q)+u_{\alpha}(t) \varphi_{\alpha}(p, q)
$$

where $H_{C}(p, q)$ is the canonical Hamiltonian and $u_{\alpha}$ are the Lagrange multipliers. According to Dirac's gauge fixing prescription, one can introduce the new "gauge " constraints

$$
\chi_{\alpha}(p, q)=0
$$

with the requirement

$$
\operatorname{det}\left\|\left\{\chi_{\alpha}(p, q), \varphi_{\beta}(p, q)\right\}\right\| \neq 0 .
$$

The maintenance of auxiliary conditions (2.6) in time gives the set of equations

$$
\dot{\chi}_{\alpha}=\left\{\chi_{\alpha}, H_{C}\right\}+\sum_{\beta}\left\{\chi_{\alpha}, \varphi_{\beta}\right\} u_{\beta}=0
$$

which allows to determine the unknown Lagrange multipliers. Formally, the solution can be written as

$$
u_{\alpha}=-\sum_{\beta} \Delta_{\alpha \beta}^{-1}\left\{H_{C}, \chi_{\beta}\right\}
$$

where $\Delta^{-1}$ is the inverse matrix of

$$
\Delta_{\alpha \beta}=\left\{\chi_{\alpha}, \varphi_{\beta}\right\}, \quad \Delta_{\alpha \beta} \Delta_{\beta \gamma}^{-1}=\delta_{\alpha \gamma}
$$

The main idea of introduction the new constraints (2.6) into the theory was to eliminate from consideration the complicated constraints (2.3), i.e. to consider them as strong equations. This result can be achieved if we pass from the Poisson brackets to Dirac's ones

$$
\begin{gathered}
\{F, G\}_{D} \equiv\{F, G\}-\left\{F, \xi_{s}\right\} C_{s s^{4}}^{-1}\left\{\xi_{s^{\prime}}, G\right\}, \\
\xi_{s} \equiv\left(\varphi_{1}, \ldots, \varphi_{m}, \chi_{1}, \ldots, \chi_{m}\right), \quad C_{\alpha \beta} \equiv\left\{\xi_{\alpha}, \xi_{\beta}\right\}, \quad C_{\alpha \beta} C_{\beta \gamma}^{-1}=\delta_{\alpha \gamma}
\end{gathered}
$$

From (2.10) one can observe that all constraints including the gauge one have zero Dirac's brackets with everything and thus we can consider them as strong equations. As it has been mentioned in the introduction, although the choice of gauge constraints allows one to take into account in an explicit form the constraint nature of canonical variables via Dirac's bracket but this gauge fixing does not provide an explicit representation for the physical phase space. We will deal with the explicit representation for the reduced phase space if one can find the conjugate coordinates $Q_{i}^{*}, P_{i}^{*}(i=1, \ldots, n-m$, so that all constraints would vanish identically as functions of these variables $\varphi_{\alpha}(p, q) \equiv \bar{\varphi}_{\alpha}\left(Q_{i}^{*} P_{i}^{*}\right) \equiv 0$ [3]. In this case, for any function $F(p, q)$ given on the reduced phase space

$$
\left.F(p, q)\right|_{\varphi=0 \chi=0}=\bar{F}\left(P^{*} Q^{*}\right)
$$


the Dirac bracket looks like the Poisson bracket for a usual unconstrained system

$$
\left.\{F, G\}_{D}\right|_{\varphi=0 \chi=0}=\sum_{i=1}^{n-m}\left\{\frac{\partial \bar{F}}{\partial Q_{i}^{*}} \frac{\partial \bar{G}}{P_{i}^{*}}-\frac{\partial \bar{F}}{\partial P_{i}^{*}} \frac{\partial \bar{G}}{Q_{i}^{*}}\right\}
$$

However, it is not easy to find these coordinates and in general one retains all dynamical variables. The change of their Poisson brackets reflects the reduction in number of the degrees of freedom

$$
\sum_{i=1}^{n}\left\{q_{i}, p_{i},\right\}_{P . B .}=n, \quad \sum_{i=1}^{n}\left\{q_{i}, p_{i},\right\}_{D}=n-m
$$

Thus, the question of "true dynamical degrees" is again open.

\section{B.2 Faddeev's scheme with constraint resolution}

In the well - known paper by L.D. Faddeev [28] the scheme of explicit reduction of phase space with the goal to extend the method of path integral quantization to a gauge theory was developed. Here we will stress only the main points of this scheme. As in Dirac's methods, we introduce the constraints

$$
\chi_{\alpha}(p, q)=0
$$

in such a way that the requirement (1.1) is fulfilled with an additional property

$$
\left\{\chi_{\alpha}(p, q), \chi_{\beta}(p, q)\right\}=0 .
$$

Now, in accordance with this property there is a canonical transformation to new coordinates

$$
\begin{aligned}
q_{i} & \mapsto Q_{i}=Q_{i}\left(q_{i}, p_{i}\right) \\
p_{i} & \mapsto P_{i}=P_{i}\left(q_{i}, p_{i}\right)
\end{aligned}
$$

such that $m$ of the new $P$ 's are

$$
P_{\alpha}=\chi_{\alpha}\left(q_{i}, p_{i}\right)
$$

The corresponding conjugate variables $Q_{\alpha}$ can be expressed with the help of the resolution of constraints (2.3)

$$
Q_{\alpha}=Q_{\alpha}\left(Q^{*}, P^{*}\right)
$$

via the $n-m$ cannonical pairs $\left(Q_{1}^{*}, P_{1}^{*}, \ldots, Q_{n-m}^{*}, P_{n-m}^{*}\right)$. This is possible due to the (1.1). This remaining variables $\left(Q_{1}^{*}, P_{1}^{*} \ldots, Q_{n-m}^{*}, P_{n-m}^{*}\right)$ spanned the $2(n-m)$ - dimensional surface $\Sigma$ determined by the equations

$$
\begin{aligned}
P_{\alpha} & =0 \\
Q_{\alpha} & =Q_{\alpha}\left(Q^{*}, P^{*}\right)
\end{aligned}
$$

After this the main point is to prove that the surface $\Sigma$ coincides with the true reduced phase space $\Gamma^{*}$ independently of the choice of gauge fixing condition. It is very attractive to 
determine the reduced phase $\Gamma^{*}$ without any gauge fixing and then to compare it with the reduce phase space obtained by Faddeev's gauge fixing method. In the next section we will describe two schemes of reduction of phase space without exploiting gauge fixing functions, solely in internal terms of the theory.

\section{Construction of the reduced phase space without gauge fixing via the "generalized canonical transformation"}

\section{C.1 Abelian constraints}

\section{i. Levi - Civita's method of reduction of systems in involution}

For explanation of main ideas of construction of the reduced subspace $\Gamma^{*}$ without using gauge fixing condition i.e. in the gaugeless manner let us first consider the special case when there are only Abelian constraints in the theory

$$
\left\{\varphi_{\alpha}(p, q), \varphi_{\beta}(p, q)\right\}=0
$$

In this case a difficulty does not arise because there is a general method of reducing in order of differential equation in the canonical if some invariant relations in involution are known [17], [18], [19]. According to Levi - Civita's method, one can perform the canonical transformation in the phase space to the new coordinates 1

$$
\begin{aligned}
q_{i} & \mapsto Q_{i}=Q_{i}\left(q_{i}, p_{i}\right) \\
p_{i} & \mapsto P_{i}=P_{i}\left(q_{i}, p_{i}\right)
\end{aligned}
$$

such that $m$ of the new $P$ 's $\left(\bar{P}_{1}, \ldots, \bar{P}_{m}\right)$ become equal to the constraints $(2.16)$

$$
\bar{P}_{\alpha}=\varphi_{\alpha}\left(q_{i}, p_{i}\right)
$$

while the remaining $n-m$ pairs of the new canonical coordinates $\left(Q_{1}^{*}, P_{1}^{*} \ldots\right.$,

$\left.Q_{n-m}^{*}, P_{n-m}^{*}\right)$ will be gauge invariant physical variables.

In terms of new canonical pairs $P, Q$ it is very useful to establish the general structure of the canonical Hamiltonian. The maintenance of complete system of irreducible constraints (2.16) in time means that

$$
\left\{\varphi_{\alpha}(p, q), H_{C}(p, q)\right\}=g_{\alpha \beta}(p, q) \varphi_{\beta}(p, q)
$$

Eq. (2.19) in the new coordinates $P, Q$ becomes

$$
\frac{\partial \bar{H}_{C}(P, Q)}{\partial \bar{Q}_{\alpha}}=\bar{g}_{\alpha \beta}(P, Q) \bar{P}_{\beta}
$$

\footnotetext{
1 Note, that in the case of the theory with reparametrization invariance we have to exploiti a more general transformation with an explicit time dependence. But in this article we restrict ourselves only to the case of gauge invariant theories.
} 


$$
\bar{H}_{C}(P, Q)=H_{C}(p(P, Q), q(P, Q))
$$

From this equation it follows that the canonical Hamiltonian $H_{C}(p, q)$, rewritten in the new coordinates $P, Q$ has the following form

$$
\bar{H}_{C}(P, Q)=\bar{H}_{0}\left(Q^{*}, P^{*}, \bar{P}\right)+\bar{\Psi}_{\alpha}(Q, P) \bar{P}_{\alpha}
$$

with some function $H_{0}\left(Q^{*}, P^{*}, \bar{P}\right)$ which does not depend on the ignorable coordinate $\bar{Q}$ :

$$
\left\{\bar{P}_{\alpha}, \bar{H}_{0}(P, Q)\right\}=0 \quad \Longrightarrow \quad\left\{\varphi_{\alpha}(p, q), H_{0}(p, q)\right\}=0
$$

and thus represents the gauge invariant part of canonical Hamiltonian. The functions $\bar{\Psi}_{\alpha}(Q, P)$ are determined through the functions $\bar{g}_{\alpha \beta}(P, Q)$ according to the equation

$$
\frac{\partial \bar{\Psi}_{\gamma}(P, Q)}{\partial \bar{Q}_{\alpha}}=\bar{g}_{\alpha \gamma}(P, Q)
$$

This property in the initial coordinates $p, q$ means that, the canonical Hamiltonian looks as follows:

$$
H_{C}(p, q)=H_{0}(q, p)+\Psi_{\alpha}(p, q) \varphi_{\alpha}(p, q)
$$

whith the gauge invariant function $H_{0}(p, q)$

$$
\left\{H_{0}(p, q), \varphi_{\alpha}(p, q)\right\}=0
$$

and functions $\Psi_{\alpha}(p, q)$ connected with $\bar{\Psi}_{\alpha}(Q, P)$ as

$$
\Psi_{\alpha}(p, q)=\bar{\Psi}_{\alpha}(P(p, q), Q(p, q))
$$

Eq.(2.23) rewritten in canonically invariant form looks like

$$
\left\{\varphi_{\alpha}(p, q), \Psi_{\gamma}(p, q)\right\}=g_{\alpha \gamma}(p, q)
$$

One would like to note that the simple definition of invariant part of canonical Hamiltonian in terms of special coordinates

$$
H_{0}(P, Q)=\bar{H}_{C}(P, Q)-\frac{\partial \bar{H}_{C}}{\partial \bar{P}_{\alpha}} \bar{P}_{\alpha}
$$

in the old coordinates can be written only through the variational derivative

$$
H_{0}(p, q)=\left[H_{C}(p, q)-\frac{\delta H_{C}}{\delta \varphi_{\alpha}} \varphi_{\alpha}\right]
$$

According to the Dirac, the time evolution of a singular hamiltonian system is governed by the extended Hamiltonian (2.5)

$$
\begin{aligned}
\dot{q}_{i} & =\left\{q_{i}, H_{E}\right\} \\
\dot{p}_{i} & =\left\{p_{i}, H_{E}\right\}
\end{aligned}
$$


In the new special coordinates instead of these equations we have the following factorazible form of canonical equations for two type of variables $P_{i}^{*}, Q_{i}^{*} \quad(i=1, \ldots, n-m)$ and $\bar{P}_{\alpha}, \bar{Q}_{\alpha}(\alpha=1, \ldots, m):$ [19], [20]

$$
\begin{aligned}
\dot{Q}_{i}^{*} & =\left\{Q_{i}^{*}, H_{P h}\right\} \\
\dot{P}_{i}^{*} & =\left\{P_{i}^{*}, H_{P h}\right\} \\
\dot{\bar{P}}_{\alpha} & =0 \\
\dot{\bar{Q}}_{\alpha} & =\bar{u}_{\alpha}(t)
\end{aligned}
$$

with an arbitrary functions $\bar{u}_{\alpha}(t)$. In (2.31) the physical Hamiltonian is defined as

$$
H_{P h}(P, Q)=\left.H_{0}\left(Q^{*}, P^{*}, \bar{P}\right)\right|_{\bar{P}=0}
$$

Thus, $\bar{Q}_{\alpha}$ are ignorable coordinates with the corresponding vanishing momenta $\bar{P}_{\alpha}$, and the canonical system allows the separation of the phase space coordinates into the physical sector and the nonphysical one

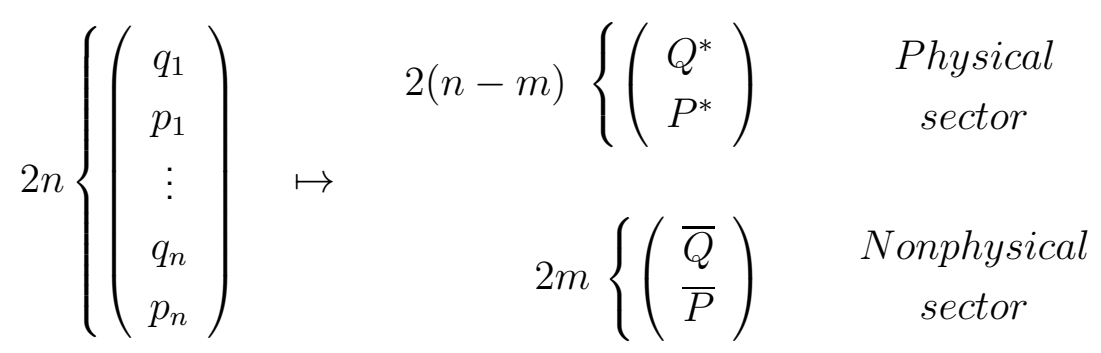

One would like to note that the choice of special canonical coordinates $P^{*}, Q^{*}$ and $\bar{Q}$ is not a unique. It is a wide freedom to define them, for example one can pass to new canonical variables

$$
\begin{aligned}
{\overline{P^{\prime}}}_{\alpha} & =\bar{P}_{\alpha} \\
\overline{Q^{\prime}}{ }_{\alpha} & =\bar{Q}_{\alpha}+f_{\alpha}\left(Q^{*}\right) \\
P_{i}^{\prime *} & =P_{i}^{*}+\bar{P}_{\alpha} \frac{\partial f_{\alpha}\left(Q^{*}\right)}{\partial Q_{i}^{*}} \\
Q^{\prime *} & =Q_{i}^{*},
\end{aligned}
$$

but in any case the above redefinition corresponds to the canonical transformation on the physical phase space spanned by the $\left(Q^{*}, P^{*}\right)$.

\section{C.2 Non - Abelian constraints}

If there are in the theory a non - Abelian constraints, the above - described procedure does not work and it is necessary to modify it. Fortunately, there is the significant observation that 
allows us to lead this case to the previous Abelian one. It can be note that in contrast with unconstrained systems the singular theories possess a wider freedom in the choice of canonical variables [30]. The canonical group of transformation for a usual unconstrained theory in this case is enlarged to the group of so - called " generalized canonical transformations " According to the definition, the generalized canonical transformations are those preserving the form of all constraints of the theory as well as the canonical form of the equations of motion [30]. The main point of our idea is to use this freedom of formulation and to pass from non - Abelian theory to an equivalent description of singular system with Abelian constraints via the generalized canonical transformation. The usual canonical transformation of variables could not change the value of the Poisson brackets, but as we will demonstraed below the transformation to new Abelian constraints is not canonical but generalized canonical transformation. In this section, we will consider two schemes of realization of this program based on the resolution of constraint or without it.

\section{i. Construction of physical coordinates via the constraint resolution}

The direct way to pass to new constraints that are Abelian and simultaneously are equivalent to the old one ( abelianization ) is as follows [21], [25]. Under the assumption that $\varphi_{\alpha}(p, q)$ are $m$ independent functions one can resolve the constraints (2.3) for $m$ of $p$ 's

$$
p_{\alpha}=F_{\alpha}(\underline{p}, q)
$$

where $\underline{p}$ denotes the remaining $p$ 's. One can now define the new equivalent to $\varphi_{\alpha}(p, q)$ constraints

$$
\Phi_{\alpha}(p, q)=p_{\alpha}-F_{\alpha}(\underline{p}, q)
$$

Now, on the one hand, by explicitlly computing one can convince onself that the Poisson brackets $\left\{\Phi_{\alpha}(p, q), \Phi_{\beta}(p, q)\right\}$ of the new constraints are independent of $p_{\alpha}$, but on the another hand, they are again the first class ones; so their Poisson brackets with each other must vanish identically. Thus after a transfomation to new constraints $\Phi_{\alpha}(p, q)$ we are ready to realize

the above mentioned canonical transformation (2.17) such that $m$ of the new $P$ 's become equal to the modified constraints $\Phi_{\alpha}(2.36)$

$$
\bar{P}_{\alpha}=\Phi_{\alpha}\left(q_{i}, p_{i}\right)
$$

with the corresponding conjugate ignorable coordinates $\bar{Q}_{\alpha}$.

\section{i.i. Abelianization of constraints via Dirac's transformation}


There is another method of abelianization without using a non painless, in general procedure of resolution of constraints against some momenta. In the previous article [32 it was shown how due to the freedom in the representation of the constraint surface $\Gamma_{c}$ defined by

$$
\varphi_{\alpha}(p, q)=0
$$

with

$$
\left\{\varphi_{\alpha}(p, q), \varphi_{\beta}(p, q)\right\}=f_{\alpha \beta \gamma}(p, q) \varphi_{\gamma}(p, q) .
$$

one can always pass with the help of Dirac 's transformation (which belongs to the class of generalized canonical transformations) ¿from these first class non - Abelian constraints to the equivalent ones

$$
\Phi_{\alpha}(p, q)=\mathcal{D}_{\alpha \beta}(p, g) \varphi_{\beta}(p, q)
$$

so that new constraints are Abelian.

$$
\left\{\Phi_{\alpha}(p, q), \Phi_{\beta}(p, q)\right\}=0
$$

As it has been mentioned above, the existences of a such a set of equivalent constraints, which can be treated as some coordinates in the manifold, is known. The question is how, in a constructive way to find this transformation matrix. According to (2.39), the matrix $\mathcal{D}_{\alpha \beta}$ must satisfy the set of the nonlinear differential equations

$$
\left\{\mathcal{D}_{\alpha \gamma}(p, g) \varphi_{\gamma}(p, q), \mathcal{D}_{\beta \sigma}(p, g) \varphi_{\sigma}(p, q)\right\}=0 .
$$

The statement of abelianization means a possibility of finding a particular solution for these very complete nonlinear differential equations. Beyond question eq. (2.40) in this form is not of any practical value; but it has been shown [32] that there is a particular solution to this equation and it can be represented as

$$
\mathcal{D}=\underbrace{\mathcal{D}^{1}(p, q) \cdots \mathcal{D}^{m}(p, q)}_{m}
$$

where each matrix $\mathcal{D}^{k}$ is again represented in product form of $k^{\prime} \mathrm{s} m \times m$ matrices

$$
\mathcal{D}^{k}=\mathcal{R}^{a_{k}+k}(p, q) \prod_{i=k-1}^{0} \mathcal{S}^{a_{k}+i}(p, q)
$$

$\left(a_{k} \equiv k(k+1) / 2\right)$ and

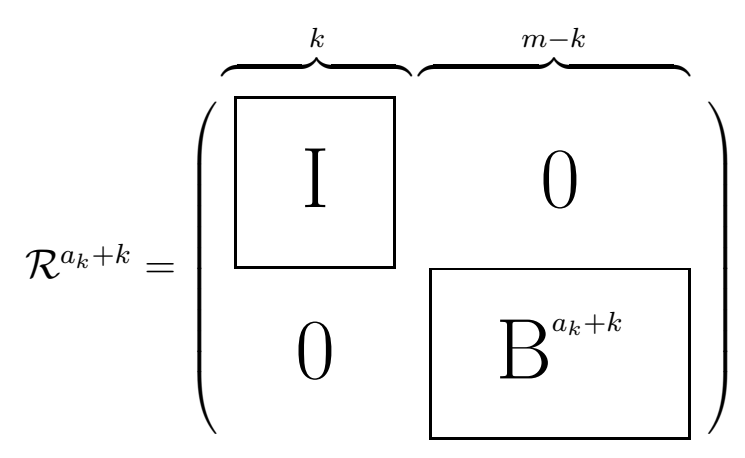




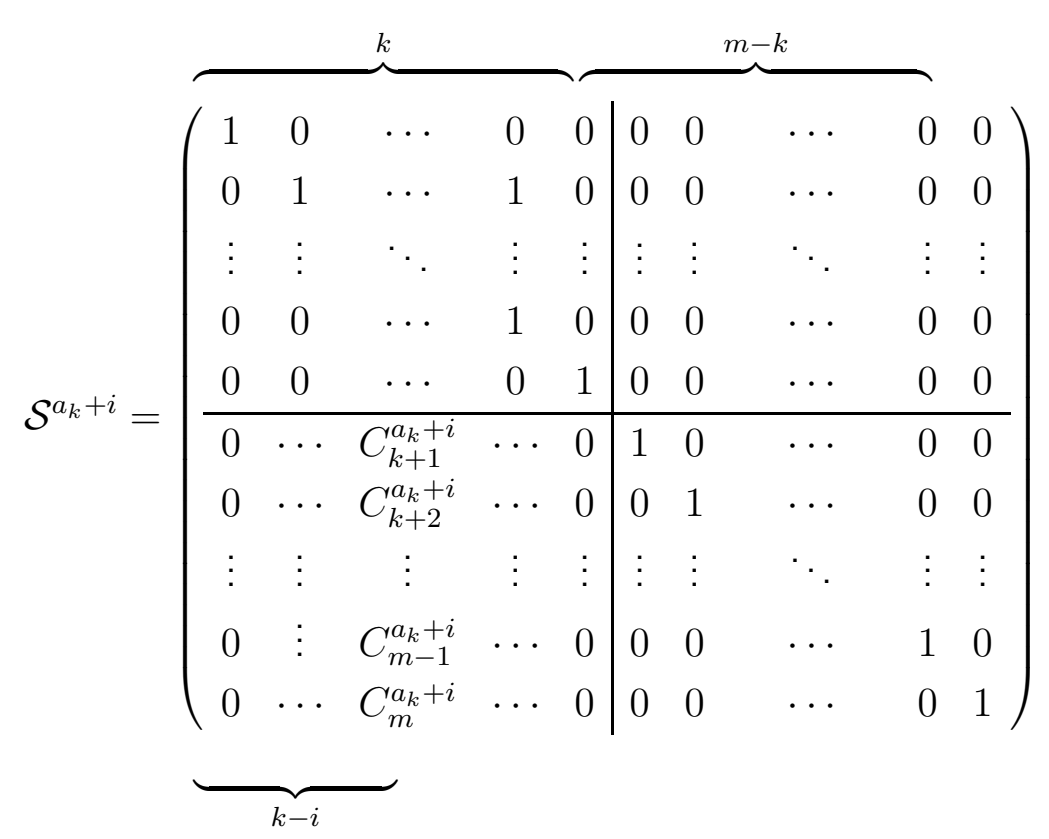

and satisfies a set of linear differential equations ( see below (2.48), (2.49)). Just the linear character of these equations allows one to speak about a practical use of the proposed method of abelianization. The constraints which are obtained as a result of the action of $k$ 's matrices (constraints at the $a_{k}+k$-th step )

$$
\Phi_{\alpha}^{a_{k}+k}=(\underbrace{\mathcal{D}^{k} \cdot \mathcal{D}^{k-1} \cdots \mathcal{D}^{k}}_{m})_{\alpha \beta} \Phi_{\beta}^{0}
$$

obey the algebra where $k$ constraints have zero Poisson brackets with any one. From the algebraic standpoint this method of abelianization represents an iterative procedure of constructing of "equivalent" algebras $\mathcal{A}^{a_{i}}$ of constraints $\Phi_{\alpha}^{a_{i}}$

$$
\mathcal{A}^{0} \underbrace{\stackrel{\mathcal{S}^{1}}{\rightarrow} \mathcal{A}^{1} \stackrel{\mathcal{R}^{2}}{\longrightarrow}}_{\mathcal{D}^{1}} \mathcal{A}^{2} \underbrace{\stackrel{\mathcal{S}^{3}}{\rightarrow} \mathcal{A}^{3} \stackrel{\mathcal{S}^{4}}{\rightarrow} \mathcal{A}^{4} \stackrel{\mathcal{R}^{5}}{\rightarrow}}_{\mathcal{D}^{2}} \mathcal{A}^{5} \ldots \underbrace{\stackrel{\mathcal{S}^{a_{k}}}{\rightarrow} \mathcal{A}^{a_{k}} \ldots \stackrel{\mathcal{R}^{a_{k}+k}}{\rightarrow}}_{\mathcal{D}^{k}} \mathcal{A}^{a_{k}+k} \ldots
$$

The abelianization procedure consists in $a_{m}$ 's steps for construction of the $m$ - dimensional abelian algebra equivalent to the initial non - Abelian one in such a manner that at the $a_{k}$ - th step the obtained algebra $\mathcal{A}^{a_{k}}$ possesses a center with $k$ elements $\mathcal{Z}_{k}[A]=\left(\Phi_{1}^{a_{k}}, \Phi_{2}^{a_{k}}, \ldots \Phi_{k}^{a_{k}}\right)$ The matrix $\mathcal{D}^{k}$ converts the algebra $\mathcal{A}^{k}$ to the algebra $\mathcal{A}^{k+1}$ in which the center contains one element more than previous.

The validity of the representation (2.42) with the matrices $\mathcal{S}$ and $\mathcal{R}$ was proved in 32 by induction. It has been shown that if $\Phi_{\alpha}^{a_{k}}$ - are constraints (obtained as result of action of the $k-1$ matrices $\left.\mathcal{D}^{i}\right)$ with the algebra having the center $\mathcal{Z}_{k}[A]=\left(\Phi_{1}^{a_{k}}, \Phi_{2}^{a_{k}}, \ldots, \Phi_{k}^{a_{k}}\right)$, then a matrix $\mathcal{D}^{k}$ from (2.41) performs the transformation to the new constraints

$$
\Phi_{\alpha}^{a_{k+1}-1}=\mathcal{D}^{k}{ }_{\alpha \beta} \Phi_{\beta}^{a_{k}+1}
$$


which form the algebra with the center $\mathcal{Z}_{k+1}[A]=\left(\Phi_{1}^{a_{k+1}}, \Phi_{2}^{a_{k+1}}, \ldots, \Phi_{k}^{a_{k+1}}\right.$, $\left.\Phi_{k+1}^{a_{k+1}}\right)$ if the matrices $\mathcal{S}$ and $\mathcal{R}$ are the solutions to the following set of linear differential equations

$$
\begin{aligned}
& \left.\begin{array}{rrr}
\left\{\Phi_{1}^{a_{k}+i-1}, S_{\alpha_{k}}^{a_{k}+i}\right\} & = & 0 \\
\vdots & \vdots & \vdots \\
\left\{\Phi_{k-1}^{a_{k}+i-1}, S_{\alpha_{k}}^{a_{k}+i}\right\} & = & 0
\end{array}\right\} \Longrightarrow\left\{\Phi_{\bar{\alpha}_{k}}^{a_{k}+i-1}, S_{\alpha_{k}}^{a_{k}+i}\right\}=0 \\
& \left\{\Phi_{k}^{a_{k}+i-1}, S_{\alpha_{k}}^{a_{k}+i}\right\}=f_{k \alpha_{k} \gamma_{k}}^{a_{k}+i-1} S_{\gamma_{k}}^{a_{k}+i}-f_{k \alpha_{k} i+1}^{a_{k}+i-1} \\
& \left.\begin{array}{rrc}
\left\{\Phi_{1}^{a_{k}+k-1}, B_{\alpha_{k} \beta_{k}}^{a_{k}+k}\right\} & = & 0 \\
\vdots & \vdots & \vdots \\
\left\{\Phi_{k-1}^{a_{k}+k-1}, B_{\alpha_{k} \beta_{k}}^{a_{k}+k}\right\} & = & 0
\end{array}\right\} \Longrightarrow\left\{\Phi_{\bar{\alpha}_{k}}^{a_{k}+k-1}, B_{\alpha_{k} \beta_{k}}^{a_{k}+k}\right\}=0 \\
& \left\{\Phi_{k}^{a_{k}+k-1}, B_{\alpha_{k} \beta_{k}}^{a_{k}+k}\right\}=-f_{k \gamma_{k} \beta_{k}}^{a_{k}+k-1} B_{\alpha_{k} \gamma_{k}}^{a_{k}+k}
\end{aligned}
$$

where $\alpha_{k}=k+1, \ldots, m, \bar{\alpha}_{k}=1,2, \ldots, k-1$ and $f_{\alpha \gamma \beta}^{a_{k}+i}$ are the structure functions of the constraints algebra $A^{a_{k}+i}$ at the $a_{k}+i$-th step.

\section{i.i.i. Construction of physical coordinates via Dirac's transformation}

However, for our purpose, to construct the coordinates of the physical subspace we can act in a slightly different way. The proposed method of explicit realization of the reduced phase space consists on the step by step elimination of ignorable coordinates of the phase space with the help of construction of the corresponding Abelian subalgebra. This can be achieved at three steps

a) first, we obtain an equivalent to the initial algebra with central element $\varphi_{1}$

$$
\left\{\varphi_{1}(p, q), \Phi_{\beta}(p, q)\right\}=0
$$

b) next, perform the canonical transformation to a new set of coordinates so that

$$
\bar{P}_{1}=\varphi_{1}\left(q_{i}, p_{i}\right), \quad\left\{\bar{Q}_{1}, \bar{P}_{1}\right\}=1
$$

c) last, restrict to the $2 n-1$-dimensional submanifold spanned by the coordinates $\bar{Q}_{\alpha_{1}}, \bar{P}_{\alpha_{1}}$, and to the algebra $\left.\bar{\Phi}_{\alpha_{1}} \equiv \Phi_{\alpha_{1}}\right|_{\bar{P}_{1}=0}$

a) First step For determination of a new algebra with one central constraint $\varphi_{1}$ one can act as it was described in [32 : 
- exclude $\varphi_{1}$ ¿from the left hand side of eq. (2.40);

- then realize abelianization with all others

Tor achieve the first, one can perform the transformation with the matrix $\mathcal{S}^{1}$

$$
\Phi_{\alpha}^{1}=\mathcal{S}_{\alpha \beta}^{1} \varphi_{\beta}
$$

of type (2.44)

$$
\mathcal{S}^{1}=\left(\begin{array}{ccccc}
1 & 0 & 0 & \cdots & 0 \\
C_{2} & 1 & 0 & \cdots & 0 \\
C_{3} & 0 & 1 & \cdots & 0 \\
\vdots & \vdots & \vdots & \ddots & \vdots \\
C_{m} & 0 & 0 & \cdots & 1
\end{array}\right)
$$

or in the expanding form

$$
\begin{aligned}
\Phi_{1}^{1} & =\Phi_{1}^{0}=\varphi_{1} \\
\Phi_{\alpha_{1}}^{1} & =\varphi_{\alpha_{1}}+C_{\alpha_{1}}^{1} \varphi_{1}
\end{aligned}
$$

The new constraints algebra remains the algebra of first class

$$
\begin{aligned}
\left\{\Phi_{1}^{1}, \Phi_{\alpha_{1}}^{1}\right\} & =f_{1 \alpha_{1} 1}^{1} \Phi_{1}^{1}+f_{1 \alpha_{1} \gamma_{1}}^{1} \Phi_{\gamma_{1}}^{1} \\
\left\{\Phi_{\alpha_{1}}^{1}, \Phi_{\beta_{1}}^{1}\right\} & =f_{\alpha_{1} \beta_{1} \gamma_{1}}^{1} \Phi_{\gamma_{1}}^{1}+f_{\alpha_{1} \beta_{1} \gamma_{1}}^{1} \Phi_{\gamma_{1}}^{1}
\end{aligned}
$$

and the new structure functions $f_{\alpha \beta \gamma}^{1}$ are determined via the old one $f_{\alpha \beta \gamma}^{1}$ and the transformation functions $C_{\alpha_{1}}^{1}$ as follows

$$
\begin{aligned}
f_{1 \alpha_{1} 1}^{1} & =f_{1 \alpha_{1} 1}+f_{1 \alpha_{1} \gamma_{1}} C_{\gamma_{1}}^{1}+\left\{\Phi_{1}^{0}, C_{\alpha_{1}}^{1}\right\} \\
f_{\alpha_{1} \beta_{1} 1}^{1} & =\frac{1}{2}\left(f_{\alpha_{1} \beta_{1} 1}-f_{\alpha_{1} \beta_{1} \gamma_{1}} C_{\gamma_{1}}^{1}+\left\{C_{\alpha_{1}}^{1}, C_{\beta_{1}}^{1}\right\} \Phi_{1}^{0}\right)- \\
& -f_{1 \alpha_{1} 1}^{1} C_{\beta_{1}}^{1}+\left\{\Phi_{\alpha_{1}}^{0}, C_{\beta_{1}}^{1}\right\}-\left(\alpha_{1} \leftrightarrow \beta_{1}\right) \\
f_{\alpha_{1} \beta_{1} \gamma_{1}}^{1} & =f_{\alpha_{1} \beta_{1} \gamma_{1}}+C_{\alpha_{1}}^{1} f_{1 \beta_{1} \gamma_{1}}-C_{\beta_{1}}^{1} f_{1 \alpha_{1} \gamma_{1}} \\
f_{1 \alpha_{1} \gamma_{1}}^{1} & =f_{1 \alpha_{1} \gamma_{1}}
\end{aligned}
$$

One can now choose the transformation functions $C_{\beta_{1}}^{1}$ so that the Poisson bracket of first constraints $\Phi_{1}^{1}$ with all other modified constraints do not contain it

$$
\left\{\Phi_{1}^{1}(p, q), \Phi_{\alpha_{1}}^{1}(p, q)\right\}=\sum_{\gamma \neq 1} f_{1 \alpha_{1} \gamma}^{1}(p, q) \Phi_{\gamma}^{1}(p, q) .
$$

these $m-1$ requirements : $f_{1 \alpha_{1} 1}^{1}=0$ according to eq. (2.55) means that the transformation function must satisfy the following set of linear nonhomogeneous differential equations

$$
\left\{\Phi_{1}^{0}, C_{\alpha_{1}}^{1}\right\}=-f_{1 \alpha_{1} 1}+f_{1 \alpha_{1} \gamma_{1}} C_{\gamma_{1}}^{1}
$$


Note that the problem of existence of solution to such a set of equations is studied very well ( see e.g. [31] ) Suppose, we find some particular solution $C_{\alpha_{1}}^{1}$ to (2.60), then one can determine all structure functions of the modified algebra according to eq.(2.55):

$$
\begin{aligned}
f_{1 \alpha_{1} 1}^{1} & =0 \\
f_{\alpha_{1} \beta_{1} 1}^{1} & =f_{\alpha_{1} \beta_{1} 1}-f_{\alpha_{1} \beta_{1} \gamma_{1}} C_{\gamma_{1}}^{1}+\left\{C_{\alpha_{1}}^{1}, C_{\beta_{1}}^{1}\right\} \Phi_{1}^{0} \\
& +\left\{\Phi_{\alpha_{1}}^{0}, C_{\beta_{1}}^{1}\right\}+\left\{\Phi_{\beta_{1}}^{0}, C_{\alpha_{1}}^{1}\right\} \\
f_{\alpha_{1} \beta_{1} \gamma_{1}}^{1} & =f_{\alpha_{1} \beta_{1} \gamma_{1}}+C_{\alpha_{1}}^{1} f_{1 \beta_{1} \gamma_{1}}-C_{\beta_{1}}^{1} f_{1 \alpha_{1} \gamma_{1}} \\
f_{1 \alpha_{1} \gamma_{1}}^{1} & =f_{1 \alpha_{1} \gamma_{1}}
\end{aligned}
$$

Now let us again keep first constraint unchanged and perform the Dirac transformation on the remaining part of the constraints $\Phi_{\alpha_{1}}, \quad \alpha_{1}=2,3, \ldots, m$

$$
\begin{aligned}
\Phi_{1}^{2} & =\Phi_{1}^{1}=\Phi_{1}^{0}=\varphi_{1} \\
\Phi_{\alpha_{1}}^{2} & =B_{\alpha_{1} \beta_{1}}^{2} \Phi_{\beta_{1}}^{1}
\end{aligned}
$$

with the requirement that new constraints have zero Poisson brackets with the first one $\Phi_{1}^{1}$

$$
\left\{\Phi_{1}^{2}, \Phi_{\alpha_{1}}^{2}\right\}=0
$$

One can verify that this requirement means that the transformation functions $B_{\alpha_{1} \beta_{1}}$ are the solutions to the equation

$$
\left\{\Phi_{1}^{1}, B_{\alpha_{1} \beta_{1}}^{2}\right\}=-f_{1 \gamma_{1} \beta_{1}} B_{\alpha_{1} \gamma_{1}}^{2}
$$

With the help of a solution of eq. (2.67) the modified algebra has the following constraints:

$$
\begin{aligned}
f_{1 \alpha_{1} 1}^{2} & =0 \\
f_{\alpha_{1} \beta_{1} 1}^{2} & =B_{\alpha_{1} \delta_{1}}^{2} B_{\beta_{1} \sigma_{1}}^{2} f_{\delta_{1} \sigma_{1} 1}^{1} \\
f_{\alpha_{1} \beta_{1} \gamma_{1}}^{2} & =\left[\left\{B_{\alpha_{1} \delta_{1}}^{2}, B_{\beta_{1} \sigma_{1}}^{2}\right\} \Phi_{1}^{\sigma_{1}}+\left\{B_{\alpha_{1} \delta_{1}}^{2}, \Phi_{\sigma_{1}}^{1}\right\} B_{\beta_{1} \sigma_{1}}^{2}-\right. \\
& \left.-\left\{B_{\beta_{1} \delta_{1}}^{2}, \Phi_{\sigma_{1}}^{1}\right\} B_{\alpha_{1} \sigma_{1}}^{2}+B_{\alpha_{1} \kappa_{1}}^{2} B_{\beta_{1} \sigma_{1}}^{2} f_{\kappa_{1} \sigma_{1} \delta_{1}}^{1}\right]\left(B^{2}\right)_{\delta_{1} \rho_{1}}^{-1}
\end{aligned}
$$

Thus as a result of two transformations $\mathcal{D}^{1}=\mathcal{S}^{1} \mathcal{R}^{2}$ we obtain the modified algebra $\mathcal{A}^{2}$ of constraints $\Phi_{\alpha}^{2}$ with the central element $\Phi_{1}^{2}$

$$
\begin{aligned}
\left\{\Phi_{1}^{2}, \Phi_{\alpha_{1}}^{2}\right\} & =0 \\
\left\{\Phi_{\alpha_{1}}^{2}, \Phi_{\beta_{1}}^{2}\right\} & =f_{\alpha_{1} \beta_{1} 1}^{2} \Phi_{1}^{2}+f_{\alpha_{1} \beta_{1} \gamma_{1}}^{2} \Phi_{\gamma_{1}}^{2}
\end{aligned}
$$

It is to be noted that due to central element nature of $\Phi_{1}^{2}$, the structure functions obey the following property:

$$
\left\{\Phi_{1}^{2}, f_{\alpha_{1} \beta_{1} \gamma}^{2}\right\}=0
$$


So, with the help of two Dirac' s transformations we obtain an equivalent to the initial algebra with one central element

$$
\left\{\varphi_{1}(p, q), \Phi_{\alpha_{1}}(p, q)\right\}=0
$$

b) Second step Now one can note that as a result of two transformations the first constraint "commutes" with all others but it can arise with left hand side of

$$
\begin{aligned}
\left\{\Phi_{\alpha_{1}}(p, q), \Phi_{\beta}(p, q)\right\} & =c_{\alpha_{1} \beta_{1} 1}(p, q) \varphi_{1}(p, q)+ \\
& +c_{\alpha_{1} \beta_{1} \gamma_{1}}(p, q) \varphi_{\gamma_{1}}(p, q)
\end{aligned}
$$

How can one shake off this term ? The following observation can help us. One can always pass to a new canonical coordinate

$$
\begin{aligned}
q_{i} & \mapsto Q_{i}=Q_{i}(q, p) \\
p_{i} & \mapsto P_{i}=P_{i}(q, p)
\end{aligned}
$$

so that one of the new momentum will be equal to the first constraint $\varphi_{1}$

$$
\bar{P}_{1}=\varphi_{1}\left(q_{i}, p_{i}\right)
$$

In these new canonical coordinates eq. (2.73) means that the new structure functions $f_{\alpha_{1} \beta_{1} \gamma}^{2}$ do not depend on the coordinate $\bar{Q}_{1}$

$$
\left\{\bar{P}_{1}, f_{\alpha_{1} \beta_{1} \gamma}^{2}(P, Q)\right\}=0 \quad \mapsto \quad \frac{\partial f_{\alpha_{1} \beta_{1} \gamma}^{2}}{\partial \bar{Q}}=0
$$

c) Third step Let us now consider the new set of constraints obtained as follows:

$$
\left.\bar{\Phi}_{\alpha_{1}} \equiv \Phi_{\alpha_{1}}\right|_{\bar{P}_{1}=0}
$$

It is not worth noting that this transition to the new set of constraints $\left.\bar{\Phi}_{\alpha_{1}} \equiv \Phi_{\alpha_{1}}\right|_{\bar{P}_{1}=0}$ is again the Dirac transformation of type (2.38) with the matrix

$$
C_{\alpha}=\left.\sum_{k=1}^{\infty} \frac{\partial^{k} \Phi_{\alpha}^{2}}{\partial^{k} P_{1}}\right|_{P_{1}=0} \bar{P}_{1}^{k}
$$

The algebra of new constraints $\Phi_{\alpha_{1}}$ has a closed form (the right - hand side do not depend on $P_{1}$ with the structure functions

$$
\bar{f}_{\alpha_{1} \beta_{1} \gamma_{1}}^{2}=\left.f_{\alpha_{1} \beta_{1} \gamma_{1}}^{2}\right|_{P_{1}=0}
$$

depending only on the remaining part of the coordinates $\bar{Q}_{\alpha_{1}}, \bar{P}_{\alpha_{1}}$, due to the property (2.78) Thus, by these admissible manipulations we reduce our problem to the equivalent one only for $m-1$ - dimensional algebra of constraints in $2 n-2$ - dimensional phase space. We will obtain the desired physical coordinates, by acting in such a manner step by step. 


\section{Conditions for admissible gauges}

As it has been mentioned above the generalized canonical transformations [30 are the those preserving the form of all constraints of the theory as well as the canonical form of the equations of motion. Thus all forms of representation of the singular theory must be connected with each other by this a kind of transformations. This allows us to give the following definition of an admissible gauge :

A gauge is admissible if and only if there is a generalized canonical equivalence between the reduced phase space obtained by the gauge fixing method and the gaugeless one.

Sufficient condition for a gauge to be admissible consists in dependence of gauge fixing conditions only on nonphysical variables. The above described method allows us to find a sufficient condition on the gauge fixing functions to belong to the class of canonical gauges for which the equivalence between the gauge fixing method and gaugeless one is fulfilled.

How can one recognize the existence of such an equivalence and what is the necessary and sufficient condition for a gauge to belong to a class of admissible gauges.

To get the answer, we must study, the general structure of the reduced theory. Let us again return to the case of the Abelian theory or to the non - Abelian theory rewritten in the equivalent abelian form. Having represented the theory in to the form (2.33) where two sectors, physical and nonphysical, are separated, it is clear that the most suitable gauge conditions are functions depending only on the ignorable coordinates $\chi_{\alpha} \equiv \chi_{\alpha}(\bar{Q})$

$$
\begin{aligned}
& \left\{\chi_{\alpha}, Q_{i}^{*}\right\}=0, \\
& \left\{\chi_{\alpha}, P_{i}^{*}\right\}=0
\end{aligned}
$$

Gauges of this type will be called the canonical gauges. Now the question is how to reformulate this property of independence on the physical variables in the initial coordinates $p, q$. At this point it is convinient to use the fact that in virtue of the definition of the physical Hamiltonian, (2.32) the requirement of independence of a gauge on physical variables can be written in canonically invariant form as 9

$$
\left.\left\{\chi_{\beta}(p, q), H_{P h}(p, q)\right\}\right|_{\Gamma^{*}}=0
$$

In this form this condition is far from practical usage. However, one could transform it to a very simple form with the help of the Dirac bracket Indeed, in the special coordinates $Q, P$

\footnotetext{
${ }^{2}$ Certainly there is a possibility when some of the physical coordinates $Q^{*}$ do not enter in to the physical Hamiltonian due to the some global symmetry and thus are usual ignorable coordinates. As a result they break through this requirement and can present in the gauge condition $\chi$, but owing to their ignorable character they can be treated in same manner as gauge noninvariant "ignorable coordinates" $\bar{Q}$.
} 
starting from the representation (2.21) one can extract from $\bar{H}_{0} \bar{P}_{\alpha}$ - independent physical Hamiltonian and write down the following decomposition for the canonical Hamiltonian

$$
\begin{aligned}
\bar{H}_{C}(P, Q) & =\bar{H}_{0}\left(Q^{*}, P^{*}, \bar{P}\right)+\bar{\Psi}_{\alpha}(Q, P) \bar{P}_{\alpha}= \\
& =\bar{H}_{P h}\left(P^{*}, Q^{*}\right)+F_{\alpha}(Q, P) \bar{P}_{\alpha}
\end{aligned}
$$

with functions $F_{\alpha}$ determined by $H_{0}$ and $\Psi$. Now, taking into account that nor the canonical gauge nor the matrix $\bar{\Delta}_{\alpha \beta}=\left\{\chi_{\alpha}, \bar{P}_{\beta}\right\}$ depend on the physical variables, we have

$$
\begin{aligned}
\left\{\bar{\chi}_{\alpha}(\bar{Q}), \bar{H}_{C}(P, Q)\right\} & =\bar{\Delta}_{\alpha \beta}(\bar{Q}) F_{\beta}(P, Q)+\left\{\bar{\chi}_{\alpha}(\bar{Q}), F_{\beta}(P, Q)\right\} \bar{P}_{\beta} \\
\left\{\bar{\Delta}_{\alpha \beta}(\bar{Q}), \bar{H}_{C}(P, Q)\right\} & =\left\{\bar{\Delta}_{\alpha \beta}(\bar{Q}) F_{\gamma}(P, Q)\right\} \bar{P}_{\gamma}+\left\{\bar{\Delta}_{\alpha \beta}(\bar{Q}), \bar{P}_{\gamma}\right\} F_{\gamma}(P, Q)
\end{aligned}
$$

Assuming for the moment that $\left\{\bar{\Delta}_{\alpha \beta}(\bar{Q}), \bar{P}_{\gamma}\right\} \neq 0$ and excluding from (3.85) functions $F_{\gamma}(P, Q)$ one get

$$
\left.\left\{\bar{\Delta}_{\alpha \beta}(\bar{Q}), \bar{H}_{C}(P, Q)\right\}\right|_{\Gamma^{*}}=\left.\left\{\bar{\Delta}_{\alpha \beta}(\bar{Q}), \bar{P}_{\gamma}\right\} \bar{\Delta}_{\gamma \sigma}^{-1}(\bar{Q})\left\{\bar{\chi}_{\sigma}(\bar{Q}), \bar{H}_{C}(P, Q)\right\}\right|_{\Gamma^{*}}
$$

Taking into account the definition (2.11) of the Dirac bracket this conditions can be rewritten in a more attractive form

$$
\left.\left\{\bar{\Delta}_{\alpha \beta}(\bar{Q}), \bar{H}_{C}(P, Q)\right\}_{D(\bar{P}, \bar{\chi})}\right|_{\bar{P}=0, \bar{\chi}=0}=0
$$

Return to the case when $\left\{\bar{\Delta}_{\alpha \beta}(\bar{Q}), \bar{P}_{\gamma}\right\}=0$ we see that there is possibility when $(3.87)$ is satisfied by the gauge condition depending on the some physical coordinates

$$
\bar{\chi}_{\alpha}=\bar{Q}_{\alpha}+f_{\alpha}\left(Q^{*}\right)
$$

But as it was mentioned above this dependence is not a significant, by canonical transformation one can get rid of it.

Now let us try to rewrite the condition (3.87) in to the old coordinates $p, q$ and for the non-Abelian form of constraints

$$
\varphi_{\alpha}=\mathcal{D}_{\alpha \beta} \bar{P}_{\beta}
$$

Due to the well - known observation [30], [23] the Dirac bracket is generalized canonical invariant object

$$
\{\bar{F}(P, Q), \bar{G}(P, Q)\}_{D(\bar{P}, \bar{\chi})}=\{F(p, q), G(p, q)\}_{D(\varphi, \chi)}
$$

and thus instead of $(3.86)$ one can write down

$$
\mathcal{D}_{\alpha \gamma}\left\{\Delta_{\gamma \beta}(p . q), H_{C}(p, q)\right\}_{D(\varphi, \chi)}+\left.\Delta_{\gamma \beta}(p . q)\left\{\mathcal{D}_{\alpha \gamma}, H_{C}(p, q)\right\}_{D(\varphi, \chi)}\right|_{\varphi=0, \chi=0}=0
$$

Now let us prove that the matrix of abelianization depends only on the variables $\bar{P}$ and $\bar{Q}$ and thus

$$
\left.\left\{\mathcal{D}_{\alpha \gamma}, H_{C}(p, q)\right\}_{D(\varphi, \chi)}\right|_{\varphi=0, \chi=0}=0
$$


It can be verified as follows. As it is known [2], [33] the generator of gauge transformations can be represented as a sum of first class constraints in non - Abelian form

$$
G=\varepsilon_{\alpha}(q, p, t) \varphi_{\alpha}(q, p) .
$$

or with the help of abelian one

$$
G=\bar{\varepsilon}_{\alpha}(\bar{Q}, \bar{P}, t) \bar{P}_{\alpha} .
$$

It is necessary to note that in eq. (3.91) the parameters of gauge transformations $\varepsilon_{A}(\bar{Q}$, depend only the ignorable coordinates $\bar{Q}, \bar{P}$ in virtue of separable form of eqs. (2.31). According to the eq.(3.91) any gauge invariant function $I$

$$
\{I, G\}=0
$$

depends only on the variables $Q^{*}$ and $P^{*}$. The $Q^{*}, P^{*}$ compose the basis of gauge invariant variables. Therefore from eq. (3.92) with generator $G$ expressed via non - Abelian constraints and matrix of abelianization according to the (3.88)

$$
G=\bar{\varepsilon}_{\alpha}(\bar{Q}, \bar{P}, t) \mathcal{D}_{\alpha \beta}^{-1} \varphi_{\beta} .
$$

one can get for $I \equiv Q^{*}, P^{*}$

$$
\begin{aligned}
& \left\{Q_{i}^{*}, G\right\}=0 \quad \Longrightarrow \quad \frac{\partial \mathcal{D}_{\alpha \beta}^{-1}}{\partial Q_{i}^{*}}=0 \\
& \left\{P_{i}^{*}, G\right\}=0 \quad \Longrightarrow \quad \frac{\partial \mathcal{D}_{\alpha \beta}^{-1}}{\partial P_{i}^{*}}=0 .
\end{aligned}
$$

where the functional independence of constraints and nonsingularity of matrix $\mathcal{D}$, have been exploited.

This completes the proof of independence of the matrix of abelianization on the variables $P^{*}$ and $Q^{*}$ and thus finally we get the desired condition

$$
\left.\left\{\Delta_{\alpha \beta}(p, q), H_{C}(p, q)\right\}_{D}\right|_{\varphi=0, \chi=0}=0
$$

where the matrix $\Delta_{\alpha \beta}=\left\{\chi_{\alpha}, \varphi_{\beta}\right\}$ is calculated with the non - Abelian constraints.

\section{Christ and Lee model}

\section{A Abelian Christ \& Lee model}

i. Reduction without gauge fixing 
Let us consider a simple mechanical system for which one can explicitly realize the above described scheme of construction of true dynamical degrees of freedom : Christ and Lee model 26

$$
\mathcal{L}=\frac{1}{2}\left(\dot{x}_{1}^{2}+\dot{x}_{2}^{2}+y^{2}\left(x_{1}^{2}+x_{2}^{2}\right)\right)-y\left(\dot{x}_{1} x_{2}-x_{1} \dot{x}_{2}\right)-V\left(x_{1}^{2}+x_{2}^{2}\right),
$$

where $\left(x_{1}, x_{2}, y\right)$ are independent coordinates. The rank of the Hessian matrix is equal to two and thus we have one primary constraint

$$
\varphi_{1}^{1}=p_{y}=0
$$

according to the definition of a canonical momentum $p_{y}$. The corresponding total Hamiltonian is

$$
H_{T}=\frac{1}{2}\left(p_{1}^{2}+p_{2}^{2}\right)-y\left(x_{1} p_{2}-x_{2} p_{1}\right)+V\left(x_{1}^{2}+x_{2}^{2}\right)+u(t) p_{y} .
$$

From the stationarity condition of primary constraint (4.96) we get the secondary constraint

$$
\varphi_{1}^{2}=x_{1} p_{2}-x_{2} p_{1}=0
$$

It is easy to verify that ternary constraints are absent and that the constraints $\varphi_{1}^{1}$ and $\varphi_{1}^{2}$ are the first class ones

$$
\left\{\varphi_{1}^{1}, \varphi_{1}^{2}\right\}=\left\{p_{y}, x_{1} p_{2}-x_{2} p_{1}\right\}=0
$$

This means that there are gauge transformations generated by

$$
G=-\dot{\varepsilon}(t) p_{y}+\varepsilon(t)\left(x_{1} p_{2}-x_{2} p_{1}\right)
$$

These gauge transformation are nothing else but a rotation around the axis orthogonal to the plane $\left(x_{1}, x_{2}\right)$ on the angle $\varepsilon(t)$.

$$
\begin{array}{r}
x_{1}^{\prime}=x_{1}+\left\{x_{1}, G\right\}=x_{1}-\varepsilon(t) x_{2} \\
x_{2}^{\prime}=x_{2}+\left\{x_{2}, G\right\}=x_{2}+\varepsilon(t) x_{1} \\
y^{\prime}=y+\{y, G\}=y-\dot{\varepsilon}(t)
\end{array}
$$

Now let us introduce the Levi - Civitta transformation to a special set of canonical coordinates $\left(y, p_{y}\right),\left(x_{1}, p_{1}\right),\left(x_{2}, p_{2}\right) \mapsto\left(Y, P_{Y}\right),\left(R, P_{R}\right),\left(\bar{\Theta}, \bar{P}_{\bar{\Theta}}\right)$ so that the new momentum $\bar{P}_{\bar{\Theta}}$ is equal to the secondary constraint $\varphi_{1}^{2}$

$$
\begin{aligned}
Y=y, & P_{Y}=p_{y} \\
R=\sqrt{x_{1}^{2}+x_{2}^{2}}, & P_{R}=\frac{x_{1} p_{1}+x_{2} p_{2}}{\sqrt{x_{1}^{2}+x_{2}^{2}}} \\
\bar{\Theta}=\arctan \left(\frac{x_{2}}{x_{1}}\right), & \bar{P}_{\bar{\Theta}}=x_{1} p_{2}-x_{2} p_{1} .
\end{aligned}
$$


These transformations are canonical and non - singular with the inverse

$$
\begin{aligned}
y=Y, & P_{Y}=p_{y}, \\
x_{1}=R \cos \bar{\Theta}, & p_{1}=P_{R} \cos \bar{\Theta}-\frac{\bar{P}_{\bar{\Theta}}}{R} \sin \bar{\Theta}, \\
x_{2}=R \sin \bar{\Theta}, & p_{2}=P_{R} \sin \bar{\Theta}+\frac{\bar{P}_{\bar{\Theta}}}{R} \cos \bar{\Theta} .
\end{aligned}
$$

everywhere except one point $R=0$ if we suppose that $0<\bar{\Theta}<2 \pi$. In terms of these variables the total Hamiltonian has the form

$$
H_{T}=\frac{1}{2}\left(P_{R}^{2}+\frac{\bar{P}_{\bar{\Theta}}^{2}}{R^{2}}\right)-Y \bar{P}_{\bar{\Theta}}+V\left(R^{2}\right)+u_{Y} P_{Y} .
$$

Note that this form is in accordance with the general representation (2.21) with the physical Hamiltonian

$$
H_{P h}=\frac{1}{2} P_{R}^{2}+V\left(R^{2}\right)
$$

and the function $\Psi$

$$
\Psi=\left(\frac{\bar{P}_{\bar{\Theta}}}{2 R^{2}}-Y\right) \bar{P}_{\bar{\Theta}}
$$

And finally from the equations of motions

$$
\begin{aligned}
& \dot{R}=P_{R}, \\
& \dot{P}_{R}=-\frac{\partial V\left(R^{2}\right)}{\partial R}, \\
& \dot{P}_{\bar{\Theta}}=0, \quad \dot{\Theta}_{\alpha}=\bar{u}_{\Theta}(t) \\
& \dot{P}_{Y}=0, \quad \dot{Y}=\bar{u}_{Y}(t)
\end{aligned}
$$

we conclude that the transformation (4.101) alows to separate the phase space coordinates into two groups: gauge invariant $R, P_{R}, P_{Y}, \bar{P}_{\bar{\Theta}}$ and noninvariant ignorable coordinates $Y, \bar{\Theta}$. This means that we achieve reduction, and now it is enough to pass to the constraint shell (in this case, it means that we must put constraints $P_{Y}$ and $\bar{P}_{\bar{\Theta}}$ equal to zero). Thus, we get the elimination of nonphysical variables without gauge fixing only through passing to the constraint shell.

\section{i.i. Gauge fixing method: example of a nonadmissible gauge}

Now we can return to a gauge fixing scheme. Any correct reduction via gauge fixing of the considered constrained system must lead to the theory that is canonically equivalent to it. First, let us write down the canonical gauge for the system (4.95).

$$
\begin{aligned}
& \chi_{1} \equiv y=0 \\
& \chi_{2} \equiv \arctan \left(\frac{x_{2}}{x_{1}}\right)=\text { constant } .
\end{aligned}
$$


For these gauge conditions the Faddeev - Popov determinant is a constant

$$
\operatorname{det}\left\|\left\{\chi_{\alpha}, \varphi_{\beta}\right\}\right\|=1
$$

The Lagrange multipliers $u_{1}, u_{2}$, can be fixed from the requirement of the stationarity of a gauge condition under the time evolution governed by the extended Hamiltonian

$$
\begin{gathered}
H_{E}=\frac{1}{2}\left(p_{1}^{2}+p_{2}^{2}\right)-y\left(x_{1} p_{2}-x_{2} p_{1}\right)+V\left(x_{1}^{2}+x_{2}^{2}\right)+u(t){ }_{1} p_{y} .+u_{2}\left(x_{1} p_{2}-x_{2} p_{1}\right) \\
u_{1}=0 \\
u_{2}=y-\frac{x_{1} p_{2}-x_{2} p_{1}}{\sqrt{x_{1}^{2}+x_{2}^{2}}}
\end{gathered}
$$

Thus the gauge (4.111) obey condition of attainability (intersect all gauge orbits ) and fix the gauge freedom in unique way and leads to the dynamics equivalent to the dynamics obtained by gaugeless method (4.110).

Now let us consider the following gauge condition

$$
\begin{aligned}
\chi_{1} & \equiv y=0 \\
\chi_{2} & \equiv \frac{x_{1}^{2}-x_{2}^{2}}{x_{1}^{2}+x_{2}^{2}}-\left(\frac{1}{2}+\frac{A}{\sqrt{x_{1}^{2}+x_{2}^{2}}}\right)=0, \quad A>0 .
\end{aligned}
$$

One can verify that for the gauge (4.113) there is a an obstruction to fulfilling the requirement of attainability. Indeed in terms of special coordinates (4.101) these gauges look as

$$
\begin{aligned}
& \chi_{1} \equiv Y=0 \\
& \chi_{2} \equiv \cos 2 \bar{\Theta}-\left(\frac{1}{2}+\frac{A}{R}\right)=0,
\end{aligned}
$$

From (4.114) we have $0<2 \bar{\Theta} \leq \frac{\pi}{3}, \frac{5 \pi}{3} \leq 2 \bar{\Theta}<2 \pi$ and thus the nonsingularity of the Faddeev - Popov determinant on the physical submanifold $\Gamma^{*}$ is fulfilled

$$
\operatorname{det}\left\|\left\{\chi_{\alpha}, \varphi_{\beta}\right\}\right\|=-\left.2 \sin 2 \bar{\Theta}\right|_{\Gamma^{*}} \neq 0
$$

One can again fix the Lagrange multipliers $u_{1}, u_{2}$, and get the description of dynamics of reduced system but it will bee non equivalent to the dynamics obtained by gaugeless method (4.110) owing to the obvious in special coordinates restriction on physical variable $R$

$$
R>2 A
$$

Finally one could note that the proposed condition for admissible gauges (3.94) forbid the using of this type of gauges. 


\section{B Non - Abelian Christ \& Lee model}

\section{Abelianization of constraints}

In this section we will apply the above described procedure of abelianization of constraints to the well known example; non - Abelian Christ and Lee model described by Lagrangian

$$
\mathcal{L}(\mathbf{x}, \dot{\mathbf{x}}, \mathbf{y})=\frac{1}{2}(\dot{\mathbf{x}}-[\mathbf{y}, \mathbf{x}])^{2}-V\left(\mathbf{x}^{2}\right)
$$

where $\mathbf{x}$ and $\mathbf{y}$-are the three - dimensional vectors, $\left(x_{1}, x_{2}, x_{3}\right),\left(y_{1}, y_{2}, y_{3}\right)$.

It is easy to verify that except for three primary constraints

$$
\boldsymbol{\pi}=\frac{\partial \mathcal{L}}{\partial \dot{\mathbf{y}}}=0
$$

there are two independent constraints

$$
\begin{aligned}
\Phi_{1}^{0} & =x_{2} p_{3}-x_{3} p_{2} \\
\Phi_{2}^{0} & =x_{3} p_{1}-x_{1} p_{3}
\end{aligned}
$$

with the algebra

$$
\left\{\Phi_{1}^{0}, \Phi_{2}^{0}\right\}=-\frac{x_{1}}{x_{3}} \Phi_{1}^{0}-\frac{x_{2}}{x_{3}} \Phi_{2}^{0}
$$

The abelianization procedure for this simple case consist of two stages At the first step the transformation $\mathcal{S}^{1}$ reduces to the

$$
\begin{aligned}
& \Phi_{1}^{1}=\Phi_{1}^{0} \\
& \Phi_{2}^{1}=\Phi_{2}^{0}+C \Phi_{1}^{0}
\end{aligned}
$$

and equation (2.48) looks like

$$
\left\{\Phi_{1}^{0}, C\right\}=\frac{x_{2}}{x_{3}} C+\frac{x_{1}}{x_{3}}
$$

One can write down a particular solution for this equation

$$
C(x)=\frac{x_{1}}{x_{3}} \arctan \left(\frac{x_{2}}{x_{3}}\right)
$$

So, as a result of the first step we get a new algebra

$$
\left\{\Phi_{1}^{1}, \Phi_{2}^{1}\right\}=-\frac{x_{2}}{x_{3}} \Phi_{2}^{1}
$$

Now let us perform the second transformation $\mathcal{R}^{2}$

$$
\begin{aligned}
& \Phi_{1}^{2}=\Phi_{1}^{1} \\
& \Phi_{2}^{1}=B \Phi_{2}^{1}
\end{aligned}
$$


with the function $B$ that satisfies the equation of type (2.49)

$$
\left\{\Phi_{1}^{1}, B\right\}=\frac{x_{2}}{x_{3}}
$$

A particular solution for this equation reads

$$
B(x)=\ln \left(\frac{\sqrt{x_{2}^{2}+x_{3}^{2}}}{x_{3}}\right)
$$

Thus, the Abelian constraints equivalent to the initial non - Abelian ones have the form

$$
\begin{aligned}
\Phi_{1}^{2} & =x_{2} p_{3}-x_{3} p_{2} \\
\Phi_{2}^{2} & =\ln \left(\frac{\sqrt{x_{2}^{2}+x_{3}^{2}}}{x_{3}}\right)\left[\left(x_{3} p_{1}-x_{1} p_{3}\right)+\frac{x_{1}}{x_{3}} \arctan \left(\frac{x_{2}}{x_{3}}\right)\left(x_{2} p_{3}-x_{3} p_{2}\right)\right]
\end{aligned}
$$

\section{Concluding remarks}

To separate the true dynamical variables from the nonphysical ones in the classical Hamiltonian systems with first class constraints without any gauge fixing, we have developed the gaugeless approach. In this approach, the reduced phase space is constructed without gauge fixing condition using the procedure of local abelianization of constraints with the subsequent canonical transformation so that some of the new momenta which are equal to the new abelian constraints while the corresponding conjugate coordinates are ignorable (nonphysical) one. The remaining canonical pairs form the basis of the reduced phase space. We have discussed the gauge fixing and gaugeless methods for reducing the phase space of a singular system with the aim to study the problem of determination of admissible gauges. We have introduced the notion of canonical gauges as functions depending only on the nonphysical variables. It is interesting to notice that suggested condition (3.94) for a gauge to be a canonical has a simple geometric meaning. As it is know [34], the inverse of Faddeev - Popov matrix $\Delta^{-1}$ represents the element of volume of phase space $\bar{\Gamma} \equiv \Gamma \backslash \Gamma^{*}$ written in noncanonical coordinates, and thus our condition (3.94) means its conservation in the process of the time evolution.

The final goal of our consideration is the construction of the reduced phase space for the complicated non - Abelian gauge theory and gravity. This program is presently under investigation, and the current article is the first step in this direction. The application of our scheme to the SU(2) Yang - Mills will be done in separate forthcoming publication.

\section{Acknowledgments}

We are happy to acknowledge interesting and critical discussions with Professors B.M. Barbashov, A.Dubin, A.T. Filippov, A.N. Kvinikhidze, G.Lavrelashvili, V.V.Nesterenko, V.A. 
Rubakov, A. Wipf. One of us (A.M.K.) would like to thank Prof. D.Wyller for kind hospitality at the Institute for Theoretical Physics of Zurich University were part of this work was done. This work was supported in part by the Russian Foundation of Fundamental Investigations, Grant No 95-02-14411. Work of A.M.K. was partly supported by the Swiss National Foundation.

\section{References}

[1] P.A.M. Dirac, Phys. Rev. 114924 (1959).

[2] P.A.M. Dirac, Lectures on Quantum Mechanics. Belfer Graduate School of Science, (Yeshive University, New York, 1964).

[3] K. Sundermeyer Constrained Dynamics, Lecture Notes in Physics N 169,( Springer Verlag, Berlin - Heidelberg - New York, 1982).

[4] J. Goldstone, R. Jackiw, Phys. Lett. B 74, 81 (1978).

[5] V.Baluni, B. Grossman, Phys. Lett B 78, 226 (1978).

[6] A.G. Izergin, V.F. Korepin, M.E. Semenov - Tyan - Shanskii, L.D. Faddeev, Teor. Mat. Fiz. 38 , 3 (1979).

[7] V.N.Pervushin, Teor. Mat. Fiz. 45, 327 (1980); , Riv. Nuovo Cimento 8 N 10, 1 (1985).

[8] R. Jackiw, Rev.Mod.Phys.52, 661 (1980).

[9] V.V.Vlasov, V.A. Matveev, A.N. Tavkhelidze, S.Yu. Khlebnikov, M.E. Shaposhnikov, Phys. of Elem. Part. Nucl. 18, 5 (1987).

[10] E.T. Newman, C.Rovelli, Phys. Rev. Lett 69, 1300 (1992).

[11] A.M. Khvedelidze, V.N. Pervushin, Helv.Phys.Acta. 67, N6, 637 (1994).

[12] V.N. Gribov, Nucl. Phys. B 139 (1978) 1.

[13] I.M. Singer, Commun. Math. Phys. 60, (1978) 7;

Physica Scripta. 24, (1981) 817;

[14] L.D. Faddeev, A.A. Slavnov, Introduction to the Quantum Theory of Gauge Fields , (Moscow, Nauka, 1984).

[15] R. Sugano, T. Kimura, J.Phys. A, 16, 4417 (1983) 
[16] M. Lavelle, D. McMullan, Phys. Lett. B 316, 172 (1993).

[17] T. Levi-Civita and U. Amaldi, Lezioni di Meccanica razionale, (Nicola Zanichelli, Bologna, 1927).

[18] E.T. Whittaker, A Treatise on the Analitical Dynamics of Particles and Rigid bodies, (Cambridge University Press, Cambridge, 1937).

[19] S. Shanmugadhasan, J. Math. Phys 14, 677 (1973).

[20] D. Dominici, J. Gomis, J. Math. Phys 21, 2124, (1980).

[21] J.Goldberg, E.T. Newman, C.Rovelli, J. Math. Phys. 32 (1991) 2739.

[22] J.A.Schouten, W.v.d.Kulk Pfaff's Problem and its Generalization (Clarendon Press, Oxford, 1949).

[23] E.C.G. Sudarshan, N. Mukunda, Classical Dynamics: A Modern Perspective, (John Willey \& Sons, New-York, 1974).

[24] T. Maskawa H. Nakajima, Prog.Theor.Phys. 56 , 1295 (1976).

[25] M.Henneaux, C.Teitelboim Quantization of Gauge Systems (Princeton University, Princeton, NJ, 1992).

[26] N.H. Christ, T.D. Lee, Phys. Rev. 22 , (1980) 939; L.V. Prokhorov, S.V. Shabanov, Usp. Fiz. Nauk. 161, N2, 13 (1991)

[27] P.A.M. Dirac, Rev. Mod. Phys. 21392 (1949).

[28] L.D. Faddeev, Theor. Math. Phys. 1, 1 (1969).

[29] N.P. Konopleva, V.N. Popov, Gauge fields , (Moscow, Atomizdat, 1972).

[30] P.G. Bergman and I. Goldberg, Phys. Rev. 98 , 531 (1955) ; P.G. Bergman ibid. 98 , $544(1955)$

[31] R. Courant, Partial Differential Equations (John Willey \& Sons, New-York, 1960).

[32] S.A. Gogilidze, A.M.Khvedelidze, V.N. Pervushin Preprint JINR E2- 95 -131.

[33] S.A. Gogilidze, V.V. Sanadze, Yu.S Surovtsev, F.G. Tkebuchava J. of Phys. A.: Math. Gen. .28, 6509 (1994); Teor. Mat. Fiz. 102, 56, (1995).

[34] V.V. Nesterenko, Teor. Mat. Fiz. 69, 115 (1986). 